\title{
A GENERALIZATION OF STURM'S SEPARATION THEOREM
}

\author{
W. ALLEGRETTO
}

Abstract. A Sturmian separation theorem is established for elliptic-parabolic equations with minimal assumptions on the coefficients and none on the regularity of the domain. A comparison theorem for linear equations and a separation theorem for quasilinear equations are then obtained as applications.

The classical Sturm separation and comparison theorems have been extended in the past few years to include the case of nonselfadjoint partial differential equations, [1], [2], [4]. The purpose of this note is to prove and apply to such equations a Sturmian separation theorem under less stringent conditions than those previously imposed.

Let $G$ denote a bounded domain of the $n$-dimensional Euclidean space $E^{n}$. We denote, as usual, by $x=\left(x_{1}, \cdots, x_{n}\right)$ the points of $E^{n}$ and differentiation with respect to $x_{i}$ by $D_{i}$ for $i=1, \cdots, n$. We consider the operator $L$ defined by:

$$
L u=\sum_{i, j=1}^{n} a_{i j} D_{i} D_{j} u+\sum_{j=1}^{n} b_{j} D_{j} u+c u
$$

whose domain $D$ is assumed to be the set $C^{2}(G) \cap C(\bar{G})$. The coefficients $a_{i j}, b_{j}, c$ are assumed real and the matrix $\left(a_{i j}(x)\right)$ symmetric nonnegative definite for every $x \in G$. No assumption is made on the continuity or boundedness of the coefficients of $L$, or on the regularity of $\partial G$.

Theorem 1. Assume $u, f, v$ are functions in $D$, and:

(1) $L u \leqq 0$ and $u<0$ throughout $G, u \equiv 0$ on $\partial G$;

(2) $L f<0$ throughout $G$;

(3) $L v \leqq 0$ throughout $G, v(x)>0$ for some $x \in G$; then v must vanish somewhere in $\bar{G}$.

Proof. If $v$ does not vanish in $\bar{G}$, then there exist positive constants $\alpha, \beta$ such that $v>\alpha>0$ and $\beta\left(\max _{x \in G}|f|\right)<\alpha$. Clearly the function $w=v+\beta f$ satisfies $L w<0$ in $G$ and $w>0$ in $\bar{G}$. Following Protter and Weinberger, [3], we introduce the function $k$ by $k=u w^{-1}$, and by considering $L(u)=L(k w)$ we see that $k$ satisfies a differential inequality to which the maximum principle can be applied. The contradiction establishes the theorem.

Received by the editors June 30, 1969 and, in revised form, January 22, 1970.

AMS Subject Classifications. Primary 3511, 3542.

Key Words and Phrases. Sturmian separation theorem, comparison theorem, nonselfadjoint equations, elliptic-parabolic equations, quasi-linear equations. 
The separation theorem now follows for operators for which there exists a function $f$ such that $L(f)<0$. We state the following result as an example:

Proposition 2. Assume that the coefficients $c, b_{j}$ are bounded in $G$ and that one of the $a_{i i}$ satisfies $a_{i i}>\gamma>0$ in $G$ for some constant $\gamma$. Let $u$, $v$ satisfy the conditions of Theorem 1 . Then $v$ must vanish somewhere in $\bar{G}$.

Proof. It is clearly sufficient to construct the function $f$ of Theorem 1 . For simplicity and without loss of generality, assume $\bar{G}$ lies in the part of $E^{n}$ where $x_{i}>0$. Let $f(x)=-x_{i}^{m}, m$ a positive integer to be later determined. We have, for $x \in G$,

$$
L f(x)=-x_{i}^{m-2}\left(\left(m^{2}-m\right) a_{i i}+m\right. \text { (bounded terms)). }
$$

Since $a_{i i}<\gamma$ in $G, L f<0$ for sufficiently large $m$.

We remark that such a function $f$ may always be constructed if the coefficient $c$ is positive in $G$ by choosing $f \equiv-1$. We also remark that the "strong" separation theorem-i.e. that $v$ must vanish somewhere in $G$ or be a constant multiple of $u$-is false under only the above assumptions, as the following example indicates: Let $L$ be given by:

$$
L y=+\left[x^{2} y^{\prime}\right]^{\prime}-4 x y^{\prime}+2 y, \quad x \in G=(0,1),
$$

where ${ }^{\prime}=d / d x$. Let $f \equiv-1, u=x^{2}-x$. Then $L u=0, u(0)=u(1)=0$, and $L f=-2$. Thus every solution $w$, not a constant multiple of $u$, of $L w=0$ in $G$ must vanish somewhere in $\bar{G}$, but, clearly, not necessarily in $G$ as may be seen by setting $w=x^{2}$.

Several other results follow immediately from Theorem 1 and Proposition 2:

Proposition 3. Assume that $u, v \in D$ and that throughout $G$ we have $L u \leqq f, L v \leqq g$ with $f, g$ bounded functions. Furthermore, if:

(i) $u=0$ on $\partial G$, $u$ negative in $G$,

(ii) $v$ somewhere positive in $G$,

(iii) $\Delta(u, v)=\left|\begin{array}{ll}f & 0 \\ u & 0\end{array}\right| \leqq 0$ in $G$,

(iv) the coefficients of $L$ satisfy the conditions of Proposition 2, then $v$ must vanish somewhere in $\bar{G}$.

Proof. If $v$ were always positive in $\bar{G}$, we could define a new operator $L_{1}$, by:

$$
L_{1} w=\left(L-g v^{-1}\right) w, \quad w \in D .
$$

Then, 


$$
L_{1} u=\left(L-\frac{g}{v}\right) u \leqq f-g \frac{u}{v}=\frac{1}{v}\left|\begin{array}{ll}
f & g \\
u & v
\end{array}\right| \leqq 0
$$

and $L_{1} v \leqq 0$ in $G$. But as a consequence of Proposition 2, $v$ must vanish somewhere in $\bar{G}$. Contradiction.

The following separation theorem is now an immediate consequence.

Proposition 4. Assume $u, v \in D$ and $L u=0$ throughout $G, u \equiv 0$ on $\partial G, u$ nontrivial. Let $L v+d v=0$ throughout $G$ and $d \geqq 0$, bounded in $G$. Then $v$ must vanish somewhere in $\bar{G}$ if the coefficients of $L$ satisfy the conditions of Proposition 2.

We conclude this note by applying the above methods to a quasilinear equation of the form:

$$
L_{2} u=L u+c^{*}(x, u) .
$$

We assume that $c^{*}(x, \xi)$ is a continuous real valued function with domain $\bar{G} \times I$, for some real interval $I$ containing the origin and that the partial derivative $c_{2}^{*}(x, \xi)$ [i.e. $\partial c^{*} / \partial \xi$ ] exists as a continuous function in $\bar{G} \times I$. We then have

Proposition 5. Assume that for some value of $i$, the function $a_{i i}$ exceeds $\gamma>0$ in $G$ and that $b_{j}$ and $c$ are bounded in $G$ for $j=1, \cdots, n$. Furthermore assume there exists a nontrivial function $u$ of class $C^{2}(G) \cap C(\bar{G})$ such that:

(i) $L_{2} u \leqq 0, u<0$ throughout $G$, and

(ii) $u \equiv 0$ on $\partial G$.

Finally let $c_{2}^{*}(x, \xi)$ be monotonically nondecreasing as a function of $\xi$ for each $x$ in $G$, and assume $c^{*}(x, 0) \geqq 0$. If $v$ is any function of class $C^{2}(G) \cap C(\bar{G})$ such that $L_{2} v \leqq 0$ throughout $G, v>0$ at some point in $G$, then $v$ must vanish somewhere in $\bar{G}$.

Proof. If not, then by the above assumptions $v>0$ throughout $\bar{G}$. Then,

$$
L u \leqq-c^{*}(u) u-c^{*}(x, 0)
$$

and

$$
L v \leqq-c^{*}(v) v-c^{*}(x, 0)
$$

where: $\quad c^{*}(u)(x)=\int_{0}^{1} c_{2}^{*}(x, t u(x)) d t, \quad c^{*}(v)(x)=\int_{0}^{1} c_{2}^{*}(x, t v(x)) d t . \quad$ We note that $c^{*}(x, u(x)), c^{*}(x, v(x))$ are bounded functions for $x \in \bar{G}$, and that $u \equiv 0$ on $\partial G, u \leqq 0$ in $G, v>0$ in $\bar{G}$. Hence, 


$$
\Delta(u, v)=u v\left[\int_{0}^{1}\left(c_{2}^{*}(x, t v)-c_{2}^{*}(x, t u)\right) d t\right]+c^{*}(x, 0)(u-v) .
$$

Since $t v(x) \geqq t u(x)$ for every $t \in[0,1]$ and $x \in G$, we see that $\Delta(u, v) \leqq 0$ in $G$. This is a contradiction of Proposition 3.

Similar results may be proved by reversing the sign of the conditions of Proposition 5.

Finally, the author wishes to take this opportunity to acknowledge the financial support of the National Research Council of Canada, while this note was in preparation.

ADDED IN PROOF. The author recently learned that R. M. Redheffer in A Sturmian theorem for partial differential equations, Proc. Amer. Math. Soc. 8 (1957), 458-462 used the same methods to obtain Sturmian theorems for a class of nonlinear equations. The results in this note, however, are not a special case of the results of Redheffer, since Theorem 1 does not require the equation to be "weakly definite", nor are the coefficients required to be of class $C^{1}$.

\section{REFERENCES}

1. K. Kreith, Sturm theorems and positive resolvents, Trans. Amer. Math. Soc 139 (1969), 319-327.

2. M. H. Protter, A comparison theorem for elliptic equations, Proc. Amer. Math. Soc. 10 (1959), 296-299. MR 21 \#5803.

3. M. H. Protter and H. Weinberger, Maximum principles in differential equations, Prentice-Hall, Englewood Cliffs, N. J., 1967. MR 36 \#2935.

4. C. A. Swanson, A comparison theorem for elliptic differential equations, Proc. Amer. Math. Soc. 17 (1966), 611-616. MR 34 \#1663.

University of British Columbia, Vancouver, British Columbia, Canada 